\title{
Wie die Sicherheit laufen lernte
}

\section{Entwicklung der funktionalen Sicherheit in Deutschland}

Der Beitrag zeigt die bisherige Entwicklung der Anlagensicherung mit Mitteln der Prozessleittechnik (PLT) auf und gibt einen Ausblick. Mit Herausgabe der IEC 61508/11 wird das abzudeckende Risiko in Sicherheitsintegritätsstufen (SIL) angegeben, denen eine nachzuweisende Mindestzuverlässigkeit der Schutzeinrichtung zugeordnet ist. Wegen der unterschiedlichsten Einsatzbedingungen in der chemischen Industrie liegen jedoch keine verlässlichen Fehlerraten von Feldgeräten vor. Es drohte ein Paradigmenwechsel, bei dem das Rechnen Vorrang gegenüber der korrekten Auslegung erhält. In Deutschland existiert ein sehr gutes PLT-Sicherheitskonzept, das dem ingenieurmäßigen Sachverstand entspricht und sich in der Praxis außerordentlich gut bewährt hat. In Zukunft sollten daher vornehmlich betriebsbewährte Geräte aus Standardgerätelisten in standardisierten Architekturen eingesetzt werden, für die Musterrechnungen vorliegen. Diese Vorgehensweise wird zur Zeit von deutscher Seite in die internationale Normung eingebracht. Dazu ist es aber unerlässlich, dass Unternehmen - speziell von der Anwenderseite - auch bereit sind, diese Aktivitäten finanziell zu unterstützen. In den folgenden Abschnitten wird die Evolution der Sicherheitstechnik beschrieben. Von deren sinngemäßer Steinzeit über deren Mittelalter gelangt man zu deren Neuzeit, die aus den Phasen „Der aufrechte Gang“, „Die internationale Normung“ und der sich notwendigerweise anschließenden „Wiederauferstehung“ besteht.

SCHLAGWÖRTER PLT-Schutzeinrichtung / SIL / Probabilistik/Ingenieursachverstand

\section{How Safety Found its Feet - \\ The Evolution of Functional Safety in Germany}

This paper outlines the development of the field "plant safety by means of process control engineering" up to now and gives an outlook on the future. With the publication of IEC 61508/11, the risk to be covered is rated by safety integrity levels (SIL). Each SIL is related to a minimum-reliability of the safety instrumented system. However, due to the different conditions in the chemical industry, there no reliable failure-rates of field device are available. There was the danger of a paradigm change towards preferring extensive mathematical calculations instead of focusing on proper design. In Germany, a very good PLT-safety-concept exists that considers the engineer's expertise and has proven itself in practical use. In the future, only prior-use-devices from standard-device-lists should be used in standardized architectures. For these architectures, example calculations are available. These aspects are introduced to the international standardization by the German delegates right now. For that purpose, it is essential that companies - especially from the user side - support these activities financially. In the following, the evolution of safety is described. Starting with the corresponding Stone Age, going through Middle Ages, one reaches the Modern Age that consists of the phases "Upright Gait", "International Standardization", and necessarily following "Resurgence". 
PIRMIN NETTER, Infraserv Höchst

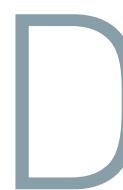
ie technische Entwicklung schreitet seit der industriellen Revolution Anfang des 19. Jahrhunderts geradezu atemberaubend voran. Als Folge von zum Teil sehr schweren Unfällen entstanden parallel dazu Sicherheits- und Unfallverhütungskonzepte. Im Bereich der Prozessindustrie wurden früher bevorzugt die unmittelbar wirksamen Schutzeinrichtungen wie Sicherheitsventile und Berstscheiben eingesetzt. Die Themen Substitution und inhärente Sicherheit folgten später.

Mit Beginn der zweiten industriellen Revolution um 1950, deren Grundlage die Rationalisierung der industriellen Fertigung durch Automatisierung ist, trat ergänzend zu den bisherigen Sicherheitssäulen eine weitere hinzu: die Anlagensicherung mit Mitteln der Automatisierungstechnik. Nach fast 30-jährigem Umgang mit dieser speziellen Art der Sicherheitstechnik möchte der Autor einen Überblick über die bisherige Entwicklung dieses wichtigen und interessanten Fachgebiets geben.

Die Anlagensicherung mit Mitteln der Automatisierungstechnik unterliegt, wie viele andere Bereiche auch, einer ständigen Evolution. Das zeigt sich rein äußerlich bereits in der Namensgebung: Ausgehend von der MSRTechnik über die EMR- oder EMSR-Technik ist man heute beim Begriff der Prozessleittechnik angelangt.

\section{STEINZEIT DER SICHERHEITSTECHNIK: 1966 BIS 1980: ERSTE GEHVERSUCHE}

Im Zuge der verstärkten Automatisierung größer und komplexer werdender Anlagen der Verfahrenstechnik in der Nachkriegszeit wurden auch der MSR-Technik in vermehrten Maße Sicherheitsaufgaben übertragen, weil die anderen Methoden in bestimmten Anwendungsfällen nicht zweckmäßig oder alleine nicht ausreichend waren.

Das erste Regelwerk, das sich mit dieser Thematik beschäftigt, ist die VDI/VDE-Richtlinie 2180 [1] aus dem Jahr 1966 mit dem Titel: „Sicherung von Anlagen der Verfahrenstechnik“. Dieses Papier wurde überwiegend von Betreibern und Herstellern erarbeitet und hatte lediglich die Anlage selbst und das erzeugte Produkt als Schutzziel im
Auge. Daher kommt auch der Name „Anlagensicherung“, der sich seltsamerweise bis heute gehalten hat, obwohl sich die Schutzziele im Sinne der Evolution deutlich ausgeweitet haben. Man sprach folgerichtig von Sicherungseinrichtungen und hat wesentliche Aspekte, die auch heute noch Gültigkeit haben, bereits damals formuliert:

- Verantwortlichkeiten sind festzulegen

- Frühzeitiges Festlegen der „Sicherheitskenngrößen“ wie Druck oder Temperatur nach den Kriterien: gut, zulässig, unzulässig

- Grenzwerte sind so zu legen, dass sie sicherheitstechnisch einwandfrei und wirtschaftlich angemessen sind

- Zweckdienliche und hochwertige Geräte mit langer Lebensdauer sind einzusetzen; Einbauort und -art sind zweckentsprechend zu wählen

- Funktionsprüfungen sollen Fehler aufzeigen, die auftreten können, aber auf andere Weise nicht erkennbar sind

- Unterscheidung in selbstmeldende und nicht selbstmeldende Ausfälle

- Eine Prüfanweisung und die Dokumentation der Prüfung wird empfohlen

- Ausführliche Behandlung der m-von-n-Bewertung

So hatte beispielsweise eine 1-von-2-Sicherungseinrichtung den in Bild 1 dargestellten Aufbau.

Wo stehen wir am Ende der Steinzeit der Sicherheitstechnik? Das Schutzziel war zwar lediglich die Anlage selbst und das erzeugte Produkt, aber mit den formulierten Konzepten war man bereits auf dem Wege zum aufrechten Gang!

\section{MITTELALTER DER SICHERHEITSTECHNIK: 1980 BIS 1993 STÖRFALLVERORDNUNG UND MIKROPROZESSOREN}

Nach dem Seveso-Unglück 1976 wurde 1980 die Störfallverordnung [2] in Kraft gesetzt. Sie fordert eine Sicherheitsanalyse, in der die sicherheitstechnisch bedeutsa- 


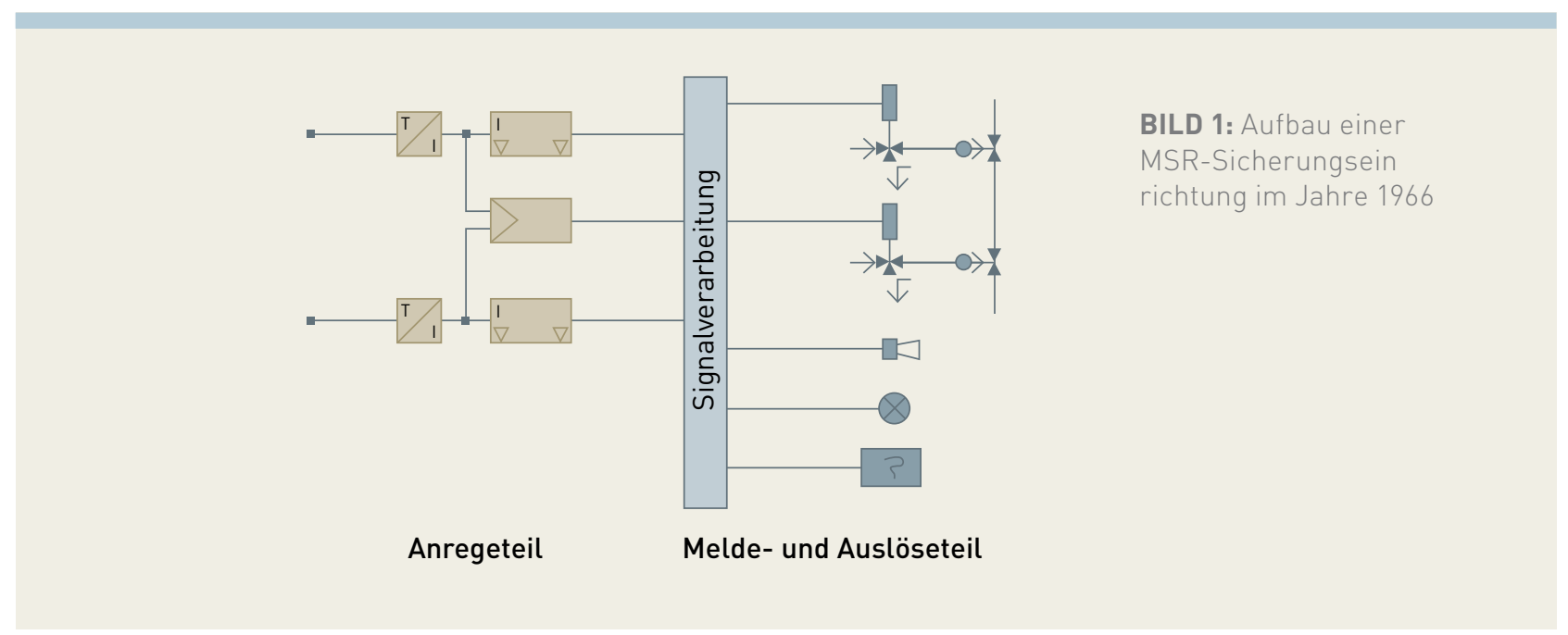

men Anlagenteile benannt werden, und verlangt Vorsorgemaßnahmen zur Verhinderung von Störfällen. Dazu gehören auch MSR-Einrichtungen und die dort so genannten Warn-, Alarm- und Sicherheitseinrichtungen. Der Widerstand gegen den damit verbundenen bürokratischen Aufwand war bei den betroffenen Firmen zum Teil erheblich. Die Dokumentationspflicht hat aber zu einer weiteren Systematisierung der Sicherheitsarbeit beigetragen.

Eine große Hilfe für Planer, Errichter und Betreiber war das 1983 erschienene Buch von G. Strohmann: „Anlagensicherung mit Mitteln der MSR-Technik“ [3]. Es enthält unter anderem eine ausführliche Diskussion des Einflusses von Gerätefehlern auf Sicherungseinrichtungen bei einund mehrkanaliger Auslegung. Es gibt kritische Anmerkungen zu der Zahlengläubigkeit in der „heutigen“ (1983!) Zeit. Quantitative Betrachtungen seien für Relativaussagen gut, für Absolutaussagen jedoch sehr fraglich.

Anfang der 80er-Jahre hält der Mikroprozessor in verstärktem Maße Einzug in die Sicherheitstechnik. Ein erstes Buch zu diesem Thema war von Hölscher/Rader: „Mikrocomputer in der Sicherheitstechnik“ [4] aus dem Jahr 1984. Hier wird dargestellt, dass komplexe Aufgaben zwar einfacher und kostengünstiger mit dieser Technik lösbar sind, das Fehlerverhalten aber nicht ohne Weiteres vorhersagbar ist. Als „Orientierungshilfe“ werden 5 Sicherheitsklassen aus den bisherigen Anwendungen in unterschiedlichen Bereichen „konstruiert“, je nach zu erwartendem Fehlerverhalten. Diesen Klassen werden Maßnahmenbündel technischer und organisatorischer Art zur Fehlervermeidung und Fehlerbeherrschung zugeordnet.

Mitte der 80er-Jahre werden weitere Schutzziele für die MSR-Technik ,entdeckt“:

\subsection{Neues Schutzziel: Personen}

Im Jahre 1984 erscheint die Zweite Auflage der VDI/VDE 2180 [5]. Die Mitglieder des Arbeitskreises waren im Wesentlichen wieder die Betreiber. Die wichtigsten Neuerungen waren:

- Klassifizierung in Betriebs- und Sicherungseinrichtungen

- Sicherungseinrichtungen werden unterteilt in Überwachungs- und Schutzeinrichtungen
- Neben den Sachschäden werden Personenschäden berücksichtigt

- Die Fehleranalyse wird verbindlich eingeführt

und „Geräte, deren Eignung durch Bewährung im praktischen Einsatz unter vergleichbaren Bedingungen erwiesen ist, sind solchen vorzuziehen, deren Eignung allein analytisch oder unter Laborbedingungen ermittelt wurde. Gleichartige Geräte für Betriebs- und Sicherungseinrichtungen innerhalb einer Anlage verringern Bedienungsfehler und erleichtern die Instandhaltung.“ [5]

Diese Aussage von damals ist heute wieder außerordentlich aktuell. Wir kommen darauf im Zusammenhang mit der NE 130 im Jahr 2010 zurück.

\subsection{Neues Schutzziel: Wasser}

Mitte der 80er-Jahre ist auch die große Zeit der Überfüllsicherungen. Das Wasserhaushaltsgesetz und länderspezifische Verordnungen (VAwS) und die TRbF 510 [6] legen Anforderungen fest, und die NE 90 interpretiert und konkretisiert die Vorgaben. Überfüllsicherungen müssen bauartzugelassen sein und regelmäßig funktionsgeprüft werden. In der Regel handelt es sich um Überwachungseinrichtungen im Sinne der VDI/VDE 2180 und nur in Ausnahmefällen um Schutzeinrichtungen. Die intensiv betriebene Umsetzung der VAwS führte zu zahlreichen Nachrüstungen in den Betrieben und zur Entwicklung neuer Überfüllsicherungen mit überwiegend aktivem Fehlerverhalten seitens der Hersteller.

\subsection{Der Risikobegriff hält Einzug}

In diesem Zeitraum gewinnt auch der Begriff des Risikos stärkere Bedeutung in der Normung. Eine klare Darstellung der Zusammenhänge zwischen Risiko, Sicherheit und Gefahr liefert die DIN VDE 31000, Teil 2 [7] durch Einführung des Grenzrisikos.

Im Jahre 1989 erscheint die DIN V 19250 [8]. Sie ist anwendungs- und technologieunabhängig und beschreibt mittels des Risikographen ein qualitatives Verfahren zur Risikoabschätzung. Es werden 8 Anforderungsklassen 
(AK) eingeführt, die ein Maß für das abzudeckende Risiko sind und die über objektivierbare Risikoparameter bestimmt werden. Den AK werden Maßnahmenbündel technischer und organisatorischer Art zugeordnet, die dann später in der DIN V 19251 [9] im Jahre 1995 ausführlich beschrieben werden. Man erkennt ansatzweise eine Orientierung am Sicherheitslebenszyklus, und es werden tiefer gehende Überlegungen, zum Beispiel anhand der Fehlertoleranzzeit, angestellt.

Leider konnte sich im AD Merkblatt A6 [10] diese „moderne Art" der sicherheitstechnischen Festlegung noch nicht durchsetzen. Als Ersatz für Sicherheitsventile werden zwar MSR-Sicherheitseinrichtungen in unterschiedlichen Auslegungsvarianten, wie fehlersicher, redundant oder selbstüberwachend vorgesehen, der Zusatz ,je nach abzudeckendem Risiko“ fehlt jedoch. Konsequenz: Noch heute werden derartige Einrichtungen grundsätzlich redundant aufgebaut.

\subsection{Papier, Papier, Papier}

... und wieder meldet sich die Störfallverordnung (Novelle 1988) [11] mit einer Erweiterung der Dokumentationspflicht für Prüf- und Überwachungsarbeiten.

\subsection{Erstes Regelwerk für Rechner}

Mit der DIN V VDE 0801 [12] werden 1990 erstmals Grundsätze für Rechner mit Sicherheitsaufgaben in einer Norm formuliert. Im Gegensatz zu konventioneller Technik liegt der Schwerpunkt der Betrachtung wegen des Softwareanteils bei den systematischen Fehlern. Auf Basis der DIN V 19250 werden Anforderungen an das Fehlerverhalten formuliert und je nach AK Maßnahmen zur Fehlervermeidung und Fehlerbeherrschung festgelegt. Die Diskussion der Fehlerarten und der Gegenmaßnahmen orientiert sich am Sicherheitslebenszyklus. Zusammen mit der Version A1 aus dem Jahr 1994 ist dieses Werk ein Vorreiter für die internationalen Normen, die im Kapitel „Neuzeit II" vorgestellt werden.

Mit Ende des Mittelalters der Sicherheitstechnik wurde also ein erheblicher Fortschritt in unserer Evolutionsgeschichte erreicht: Mit der Entdeckung von „Leib und Leben“ als Schutzziel, der Orientierung am abzudeckenden Risiko über die Anforderungsklassen, der Integration von Mikroprozessoren in die Sicherheitswelt und einer sauberen, nachvollziehbaren Dokumentation geht die Entwicklung voran.

\section{NEUZEIT DER SICHERHEITSTECHNIK, EPOCHE I: 1993 BIS 2002 - DER AUFRECHTE GANG}

Die Neuzeit bricht Anfang der 90er-Jahre an. Es war bereits sehr vieles geregelt, insbesondere durch die VDI/VDERichtlinie 2180. Es gab jedoch zwei Herausforderungen:

1 In anderen Regelwerken wurden andere Begriffe benutzt. Es gab Sicherungseinrichtungen, Sicherheitseinrichtungen, Schutzeinrichtungen, Überwachungseinrichtungen, Warn-, Alarm- und Sicherheitseinrichtungen.

2 | Historisch gewachsen entstanden verschiedene firmenspezifische PLT-Sicherheitskonzepte, die sich zwar alle stark an der VDI/VDE-Richtlinie 2180 orientierten, aber mit unterschiedlicher Terminologie: So gab es Einrichtungen für Schutz, Sicherheit, Klassen A, B, C, Kategorie I und II, Klassen A0, A1, A2 und Schadensbegrenzungseinrichtungen.

Im Zeitalter der Störfallvordnung landeten dann Sicherheitsanalysen unterschiedlicher Unternehmen auf dem Schreibtisch desselben Gutachters oder Aufsichtsbeamten. Das Erstaunen über die Vielfalt der Begriffe und Konzeptionen war bei diesen Personen verständlicherweise sehr groß. Es bestand also ein erheblicher Harmonisierungsbedarf. Dieser Aufgabe nahm sich die Namur an, und unter der Obmannschaft des Autors entstand 1993 im Namur-AK 4.5 die NE 31: ,Anlagensicherung mit Mitteln der Prozessleittechnik" [13]. Dieses Papier wurde damals zur auflagenstärksten Namur-Empfehlung. Die wesentlichen Inhalte waren:

- Erstmals Einführung des Schutzziels Umwelt (als Konsequenz aus der StörfallV)

- Klassifizierung und Kennzeichnung in:

$\square$ PLT-Schutzeinrichtungen, Klasse A: Personen- und Umweltschäden

$\square$ PLT-Schutzeinrichtungen, Klasse B: Sachschäden

$\square$ PLT-Überwachungseinrichtungen, Klasse C

$\square$ Neu! PLT-Schadensbegrenzungseinrichtungen, Klasse X

Einführung der Risikobereiche I und II für Schutzeinrichtungen, je nach abzudeckendem Risiko und sicherheitsbezogener Verfügbarkeit der eingesetzten Geräte

- Vorgehensweise bei Schutzeinrichtungen:

$1 \mid$ Abschätzung des abzudeckenden Risikos

2 | Festlegung der Anforderungen

3 | Zuordnung technischer und organisatorischer Maßnahmen

- Gegenüberstellung der Begriffe aus der NE 31 und der StörfallV

In der DIN V VDE 0801/A1 (1994) [14] wurde erstmals der Begriff „betriebsbewährt“ näher gefasst: „Einsatz einer Betrachtungseinheit, die im Wesentlichen unverändert über einen ausreichenden Zeitraum in zahlreichen, verschiedenen Anwendungen betrieben wurde und bei der keine oder nur unwesentliche Fehler festgestellt wurden (mind. 10 Systeme, je 1 Jahr Betriebsdauer).“ Darüber ist eine Dokumentation zu führen. Zweck der Vorgehensweise ist der Nachweis, dass das System hinreichend frei von systematischen (Entwurfs-)Fehlern ist.

Das VdTÜV-Merkblatt 372 [15] aus dem Jahre 1997 beschäftigt sich mit der Prüfung sicherheitsrelevanter MSREinrichtungen. Nach jeder Phase des Sicherheitslebenszyklus wird ein Haltepunkt eingeführt, und es werden die jeweilig erforderlichen Prüfschritte beschrieben.

Erfreulicherweise wurden die Empfehlungen der NE 31 in den Firmen weitgehend umgesetzt, sodass Mitte der 90er-Jahre damit begonnen werden konnte, die VDI/VDE 2180 [16] abermals zu aktualisieren. Dies erfolgte erstmals unter Einbeziehung aller am Sicherheitsprozess beteiligten Gruppen: Herstellern, Anwendern, Gutachterinstitutionen, der Wissenschaft und Aufsichtsbehörden. In der Auflage von 1998 wurden viele Aspekte der NE 31 übernommen und die StörfallV einbezogen. Daraus resultierte die klare 
Unterscheidung in die nicht sicherheitsrelevanten Betriebsund Überwachungseinrichtungen (Kennzeichnung S), die im Prozessleitsystem integriert werden dürfen, und die sicherheitsrelevanten Schutz- und Schadensbegrenzungseinrichtungen (Kennzeichnung Z), die über sicherheitsgerichtete Steuerungen realisiert werden müssen. Es wurde der Risikograph aus der DIN V 19250 übernommen, als eine Methode der qualitativen Risikoabschätzung.

Die VDI/VDE 2180 wird später (2004) in der „Vollzugshilfe zur Störfallverordnung“ "17], die die Verwaltungsvorschriften ersetzt, einbezogen und wird damit zum wichtigsten Regelwerk der funktionalen Sicherheit in Deutschland. 1999 erscheint die NE 79: „Mikroprozessorbestückte Geräte in der Anlagensicherung“ [18]. Sie ist unter Mitarbeit namhafter Hersteller entstanden. Seit Ende der 80er wurden sicherheitsgerichtete speicherprogrammierbare Steuerungen (SSPS) zertifiziert. Das wurde seitens der Anwender akzeptiert, da diese Systeme in den Betrieben vergleichbaren Einsatzbedingungen ausgesetzt sind (,,klimatisierter Schaltraum“), die wenig von den Laborbedingungen, unter denen sie getestet wurden, abweichen.

In der NE 79 wird allerdings klar festgestellt, dass die Zertifizierung von Feldgeräten, analog zu den SSPS, nicht zweckmäßig und somit auch nicht erwünscht sei, da die individuellen Prozess-, Stoff- und Umgebungsbedingungen in einem derartigen Verfahren nicht berücksichtigt werden können. Aber gerade dieses Thema, die Anpassung der am Markt erhältlichen Geräte an den eigenen Prozess, ist der Kern professioneller Sicherheitsarbeit und erfordert dringend den ingenieurmäßigen Sachverstand und umfassende Erfahrung. Deshalb wurden in der NE 79 alternativ zur Zertifizierungspraxis Wege aufgezeigt, wie man zu betriebsbewährten Geräten gelangen kann.

Die wesentlichen Inhalte sind:

- Aussagen über den Nachweis der Betriebsbewährung durch Anwender in drei Schritten:

$\square$ Anwender-Typprüfung

$\square$ Gezielte Erprobung

$\square$ Einsatz einer größeren Stückzahl über einen längeren Zeitraum (10 an unterschiedlichen Stellen mit unterschiedlichen Randbedingungen, ein Jahr lang)

- Aussagen über den Nachweis der Betriebsbewährung durch Hersteller/Vorgehensweise beim Vorliegen einer Herstellerbescheinigung (Muster dafür in der Ausgabe 2004)

- Aussagen über die Vorgehensweise bei nicht betriebsbewährten Geräten

- Anforderungen an Erst- und Wiederholungsprüfungen

- Forderungen an Hersteller für künftig zu entwickelnde Geräte

Als Hauptziel wurde formuliert: Die Anwender benötigen einen Gerätetyp, der „AK-4 fähig“ und einkanalig bis AK-4 und in 1v2-Redundanz für AK 5 und 6 einsetzbar ist.

Im Jahr 2000 erscheint Blatt 5 der VDI/VDE-Richtlinie 2180 [19] mit dem Titel „Einsatz von speicherprogrammierbaren Steuerungen“. Ebenfalls im Jahr 2000 fordert eine weitere Novelle der StörfallV die Einführung eines Sicherheitsmanagementsystems. Die Vervollständigung in dieser Epoche war die NE 93: „Nachweis der sicherheitstechnischen Zuverlässigkeit von PLT-Schutzeinrichtungen“ [20] aus dem Jahr 2001. Hiermit wurde der
Grundstein für eine flächendeckende Stördatenerfassung in Deutschland gelegt. Es wurden die Mindestanforderungen für eine derartige Datensammlung formuliert und ein Konzept erstellt, wie die Zahlen über die Namur zusammengeführt und ausgewertet werden können. Als großer Erfolg darf dabei gewertet werden, dass sich mittlerweile etwa 40 Firmen an der Stördatenerfassung beteiligen mit insgesamt rund 40000 eingesetzten PLT-Schutzeinrichtungen. Mit Hilfe dieser Daten konnte der Nachweis erbracht werden, dass die in den Regelwerken formulierten Sicherheitskonzepte in der Praxis greifen.

\section{NEUZEIT DER SICHERHEITSTECHNIK, EPOCHE II: 2002 BIS 2007 - DIE INTERNATIONALE NORMUNG SCHLÄGT ZU}

Ergänzend zum damit erreichten ,aufrechten Gang der Sicherheitstechnik" sind zwei weitere Namur-Empfehlungen entstanden:

1 | Die NE 97: „Feldbus für Sicherungsaufgaben“ [21] im Jahr 2003. Hier wird näher auf die Zertifizierungspraxis eingegangen und auf die Trennung von sicherheitsrelevanten Komponenten des Feldbusses von nicht sicherheitsrelevanten.

2 | Die NE 106: „Prüfintervalle für PLT-Schutzeinrichtungen“ [22] im Jahr 2006. In diesem Papier wird beschrieben, wie das bisher übliche Mindestprüfintervall von einem Jahr verlängert werden kann. Wesentliche Voraussetzungen dafür sind die Mehrkanaligkeit und ein hoher Grad von Diagnosemöglichkeiten.

Mit der Übernahme international entstandener Normen in das nationale Regelwerk, der DIN EN 61508 (VDE 0803) [23] im Jahr 2002 und der DIN EN 61511 (VDE 0810) [24] im Jahr 2005, verbunden mit der Zurückziehung aller nationalen Normen auf diesem Gebiet am 1. August 2004, drohte jedoch ein Paradigmenwechsel. In diesen neuen Regelwerken ist die Orientierung am Sicherheitslebenszyklus und die damit verbundene Konzentration auf das Vermeiden systematischer Fehler in der Entwicklungsphase von Geräten zu begrüßen. Die Forderung nach einem Sicherheitsmanagementsystem unterstützt die notwendige systematische Vorgehensweise bei Sicherheitsaufgaben und regelt klar die Verantwortlichkeiten. Ebenso ist die Einführung von Sicherheitsintegritätsstufen (Safety Integrity Levels), den SIL, akzeptiert. Lassen sie sich doch sehr gut auf die ehemaligen AKs abbilden. Neu in der Sicherheitsbetrachtung war der Schwenk von den einzelnen Geräten hin zur gesamten Sicherheitsfunktion. Jedem SlL wird eine Mindestzuverlässigkeit für die PLT-Schutzfunktion zugeordnet, die „Probability of Failures on Demand“ (PFD). Was auch noch heute erhebliches Kopfzerbrechen in diesem Zusammenhang bereitet, ist die Forderung eines rechnerischen Nachweises der PFD für derartige Schutzkreise. Die Formel für einkanalige Systeme lautet:

$P F D=1 / 2 \cdot \lambda_{D U} \cdot T_{i}$

Mit PFD: Wahrscheinlichkeit eines passiven Fehlers bei Anforderung

$\lambda_{D U}$ : Fehlerrate gefährlicher, unentdeckter Fehler

$T_{i}$ : Prüfintervall 
In der chemischen und pharmazeutischen Industrie liegen allerdings keine verlässlichen Daten über die Ausfallraten einzelner Geräte vor, weil diese in ganz unterschiedlichen Umgebungsbedingungen eingesetzt werden. Das betrifft insbesondere die Feldgeräte, die Sensoren und Aktoren. Die Raten können um Zehnerpotenzen schwanken! Trotzdem werden zahlreiche Rechnungen durchgeführt, bei denen das Ergebnis auf vier oder mehr Stellen hinter dem Komma genau angegeben wird. Zwei Beispiele sollen die Problematik verdeutlichen:

Das erste Beispiel zeigt, wie empfindlich das Ergebnis auf die Variation der Eingangswerte reagiert. Die Schutzeinrichtung hat die in Bild 2 gezeigte Redundanzstruktur.

Die Näherungsformel aus Blatt 4 der VDI/VDE 2180 für dieses System lautet:

$$
\begin{aligned}
P F D_{\text {total }}= & \left(\lambda_{D U}^{2} \cdot T_{i}^{2}+\beta \cdot 1 / 2 \lambda_{D U} \cdot T_{i}\right)_{\text {Sensor }}+P F D_{\text {Logik }}+ \\
& \left(\left(\lambda_{D U}^{2} \cdot T_{i}^{2}\right) / 3+\beta \cdot 1 / 2 \lambda_{D U} \cdot T_{i}\right)_{\text {Aktor }}
\end{aligned}
$$

Mit $\beta$ : Anteil unerkannter passiver Fehler gemeinsamer Ursache

Die PFD der Logik beträgt $2 \times 10^{-5}$, und für $\beta$ wird ein Wert von $5 \%$ angenommen. Aus zwei unterschiedlichen Quellen findet man für den Aktor identisches $\lambda_{A}$ von 0,05, für den gleichen Sensor jedoch unterschiedliche Werte für $\lambda_{S}$ von 0,3 beziehungsweise 0,02. Führt man die Berechnung alternativ mit diesen beiden Werten durch und variiert $T_{i}$ zusätzlich zwischen monatlich und jährlich, so erhält man 4 Fallunterscheidungen mit folgendem interessanten Ergebnis (siehe Tabelle 1).

Die Ergebnisse variieren also zwischen SIL 1 und SIL 3, je nach Wahl der Eingangsdaten.
Welche Stilblüten die Rechnerei mitunter noch hervorbringt, zeigt das 2. Beispiel, ebenfalls aus der Praxis: Eine relativ komplexe Schutzeinrichtung (siehe Bild 3), bei der jeder der fünf Zweige nochmals eine Unterstruktur hat und in der insgesamt etwa ein Dutzend Sensoren und Aktoren eingesetzt sind, wird vom Gutachter folgendermaßen beschrieben:

„Die PFD bei dieser Einrichtung ist kleiner als 6,2176 $10^{-4}$. Dieses Ergebnis entspricht SIL 3 gemäß IEC 61511. Die Eingangsparameter sind allgemein zugänglichen Quellen entnommen." Das Ergebnis wird auf vier Stellen genau angegeben. Wissenschaftliche Untersuchungen haben jedoch ergeben, dass die Eingangsparameter bei gleichen Geräten um Größenordnungen schwanken können. Bereits im ersten Semester eines Ingenieurstudiums lernt man, wie man mit einer derartigen Aufgabenstellung richtig umgeht! Auch die SIL-Zertifizierungen von Einzelgeräten werden ohne Berücksichtigung der genannten Unschärfen durchgeführt. Die Hersteller sind dabei oft auf die Angaben von Fehlerraten der verbauten Komponenten ihrer Unterlieferanten angewiesen. Mittlerweile sehen nicht nur die Anwender, sondern auch andere am Sicherheitsprozess beteiligte Gruppen die Zertifizierung von Feldgeräten als sehr fragwürdig an.

Noch problematischer ist es, aus solchen Datenquellen eine nutzbare Lebensdauer für Geräte in Sicherheitsanwendungen abzuleiten. In der DIN EN 61508 (VDE 0803) wird zum Beispiel eine mittlere Lebensdauer von 8-12 Jahren (abgeleitet von Elektrolytkondensatoren!) angegeben. Prompt wird das auf alle Geräte, sogar rein mechanische Komponenten, übertragen. Damit nicht genug! Es gibt SIL-Zertifikate, in denen Lebensdauern angegeben werden, die nur auf Annahmen beruhen und in denen die Instandhaltungsmaßnahmen der Anwender völlig außer Acht gelassen werden, mit dem Hinweis, dass nach Ablauf dieser Lebensdauer das SIL-

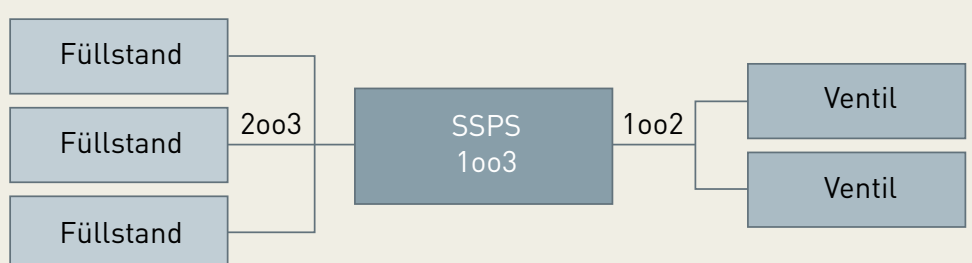

\begin{tabular}{|l|c|c|c|}
\hline Sensorik 2003 & Logik 1003 & Aktorik 1002 & Total \\
\hline $\begin{array}{l}\text { Quelle 1 9,8 } \cdot 10^{-2} \\
\text { jährliche Prüfung }\end{array}$ & $2 \cdot 10^{-5}$ & $2,1 \cdot 10^{-3}$ & $\begin{array}{c}1 \cdot 10^{-1} \\
\text { SIL } 1\end{array}$ \\
\hline $\begin{array}{l}\text { Quelle 2 } 9 \cdot 10^{-4} \\
\text { jährliche Prüfung }\end{array}$ & $2 \cdot 10^{-5}$ & $2,1 \cdot 10^{-3}$ & $\begin{array}{c}3 \cdot 10^{-3} \\
\text { SIL 2 }\end{array}$ \\
\hline $\begin{array}{l}\text { Quelle 1 1,3 10-3 } \\
\text { monatliche Prüfung }\end{array}$ & $2 \cdot 10^{-5}$ & $1,1 \cdot 10^{-4}$ & $\begin{array}{c}1,4 \cdot 10^{-3} \\
\text { SIL 2 }\end{array}$ \\
\hline $\begin{array}{l}\text { Quelle 2 4,4 10-5 } \\
\text { monatliche Prüfung }\end{array}$ & $2 \cdot 10^{-5}$ & $1,1 \cdot 10^{-4}$ & $\begin{array}{c}1,7 \cdot 10^{-4} \\
\text { SIL 3 }\end{array}$ \\
\hline
\end{tabular}

BILD 2:

Redundanzstruktur für Beispiel 1

TABELLE 1: Rechenergebnisse bei Variation der Eingangsparameter

- Quelle 1 mit jährlicher Prüfung führt zu SIL 1 - Quelle 2 mit jährlicher Prüfung zu SIL 2

- Quelle 1 und monatlicher Prüfzyklus ergibt SIL 2 und

- Quelle 2 mit monatlicher Prüfung sogar SIL 3 


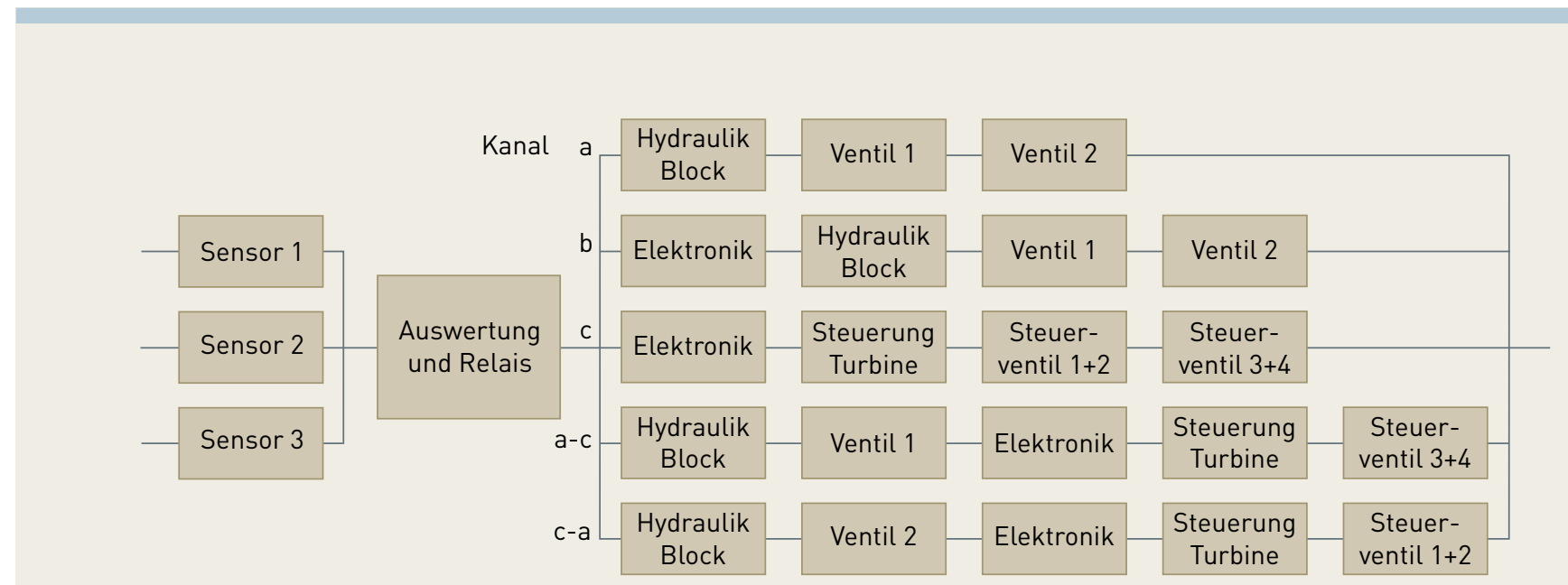

BILD 3: Blockschaltbild einer komplexen PLT-Schutzeinrichtung

Zertifikat seine Gültigkeit verliert. Abenteuerlich! Auf Basis dieser Erkenntnisse ist die Vorgehensweise einiger Anwender als sehr bedenklich anzusehen, nur noch SIL-zertifizierte Geräte einzusetzen, eine „exakte“ Berechnung des kompletten Sicherheitskreises durchführen zu lassen, die Zertifikate samt Berechnung abzuheften und dann noch zu glauben, jetzt sei das Wesentliche für die Sicherheit getan.

Einem Alibidenken wird Vorschub geleistet, nach dem Motto: „Das SIL-Zertifikat ist ja vorhanden, jetzt kann nichts mehr passieren“. Ein typischer Fall von „Schein“Sicherheit. Eine wichtige Tugend des ingenieurmäßigen Sachverstands: zu prüfen, ob die Systeme auch wirklich zum konkreten Anwendungsfall passen, droht zu verkümmern. Wer die neuen Normen so versteht und lebt, muss aufpassen, dass er nicht einen erheblichen Rückschlag in seiner Sicherheitsevolution erlebt.

\section{NEUZEIT DER SICHERHEITSTECHNIK, EPOCHE III: SEIT 2007 - WIEDERAUFERSTEHUNG}

Die Namur passt auf! Was zu Ende des vorigen Kapitels eher negativ geschildert wurde, sind Auswüchse, die hoffentlich selten vorkommen. Die neuen Normen haben durchaus auch positive Aspekte. Sie waren der Anlass, die Systematik unserer bereits ausgereiften Sicherheitskonzepte erneut auf den Prüfstand zu stellen und einige Anregungen nutzbringend einzubinden.

Zuerst galt es, die bewährte nationale Vorgehensweise formal zu „retten“. Immerhin haben sich seit Mitte der 80er-Jahre keine größeren Störereignisse aufgrund des Versagens von PLT-Schutzeinrichtungen in der Bundesrepublik ereignet. Warum sollte man dieses Know-how dann über Bord werfen? Die nationalen Normen sind zwar nicht mehr gültig, aber wer hinderte uns daran, die VDI/VDE 2180 [25] erneut zu überarbeiten und alle bewährten Konzepte aus diesen Papieren und die wesentlichen Aussagen aus den einschlägigen Namur-Empfehlungen mit einzubeziehen? Natürlich muss auch die neue international entstandene Normenwelt berücksichtigt werden. Dieser
Kraftakt war 2007 bis 2010 gelungen. Abermals haben alle an dem Sicherheitsprozess beteiligten Gruppen bei der Erarbeitung dieser vierten Auflage mitgewirkt. Diese bewährte Methode stellt sicher, dass bei der späteren Anwendung der Richtlinie deren Akzeptanz besonders hoch ist. Schließlich beschreibt die VDI/VDE 2180 den Stand der Sicherheitstechnik im Sinne der Störfallverordnung.

Die wesentlichen Neuerungen sind:

- Übernahme der SIL in den Risikographen

- Zuordnung der PFD zu den SIL

- Ausführliche Beschreibung eines Sicherheitsmanagementsystems mit Themen wie Verantwortlichkeit, Qualifikation, Dokumentation

- Musterrechnungen zum Nachweis der Hardwaresicherheitsintegrität bei betriebsbewährten Geräten (Basisdaten aus NE 93 beziehungsweise NE 130)

- Empfehlungen zur Umsetzung in die Praxis

- Schadensbegrenzungseinrichtungen heißen jetzt schadensbegrenzende Schutzeinrichtungen (analog zur Terminologie der Störfallverordnung)

Im Jahr 2009 erscheint die NE 126: „Bestandsschutz für PLT-Schutzeinrichtungen“ [26]. Sie füllt eine Lücke, die andere Regelwerke offen lassen. Nirgendwo findet man in Normen oder VDI-Richtlinien Aussagen über dieses wichtige Thema. Also hat man bei der Namur den gesunden Menschenverstand walten lassen und formuliert: „Bestehende PLT-Schutzeinrichtungen genießen Bestandsschutz, falls sie nach den bisher angewendeten Regelwerken geplant, errichtet und betrieben wurden.“ Diese Aussage wird mit Beispielen verdeutlicht.

\subsection{NE 130: betriebsbewährte Geräte}

Das neueste und besonders wichtige Werk aus dem Namur-AK 4.5 ist die Anfang 2010 erschienene NE 130: „Betriebsbewährte Geräte für PLT-Schutzeinrichtungen und vereinfachte SIL-Berechnung“ [27]. 


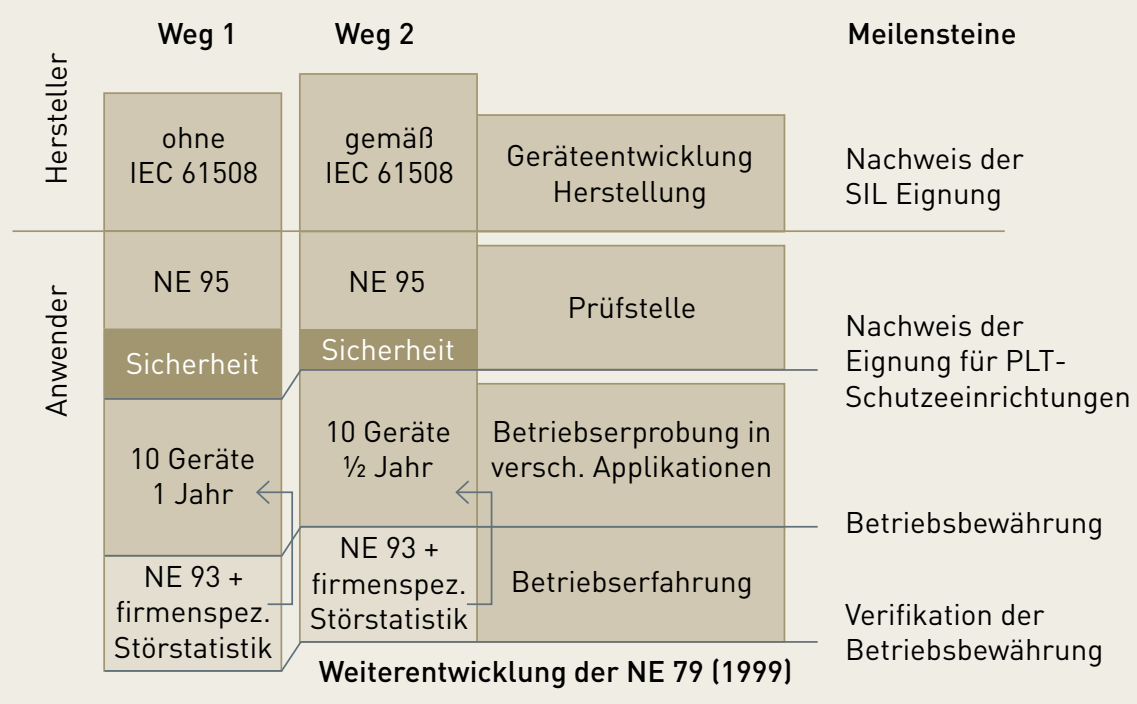

BILD 4: Prinzipielle

Darstellung des

Betriebsbewährungsprozesses

\section{Fehlerbetrachtung}

Zunächst wird in diesem Papier ausführlich der Unterschied zwischen systematischen und zufälligen Fehlern herausgearbeitet. Die DIN EN 61508 (VDE 0803) und auch das Konzept der Betriebsbewährtheit zielen darauf ab, die systematischen Fehler im Entwicklungsprozess der Geräte möglichst zu vermeiden und bei den ersten Anwendungsversuchen noch verbliebene Fehler dieser Art aufzudecken. Bei dem probabilistischen Ansatz wird davon ausgegangen, dass die systematischen Fehler bereits eliminiert sind und sich die PFD-Berechnungen lediglich auf die verbleibenden zufälligen Fehler beziehen, was von den Anwendern allzu leicht übersehen wird.

\section{Betriebsbewährung}

Das Konzept der Betriebsbewährung erfolgt in 4 Schritten (siehe Bild 4):

1 | Nachweis der SIL-Eignung

2 | Nachweis der Eignung für PLT-Schutzeinrichtungen

3 | Nachweis der Betriebsbewährung mit Betriebserprobung

4 | Verifikation der Betriebsbewährung

Das ist nicht neu (siehe NE 79 von 1999) und hat auch zunächst nichts mit den internationalen Normen zu tun. Eine Erleichterung tritt jedoch ein, wenn die Geräte bereits nach DIN EN 61508 (VDE 0803) entwickelt wurden. Umgekehrt widerspricht der Verzicht auf den Nachweis der Betriebsbewährtheit, nur weil ein SILZertifikat oder eine Herstellerbescheinigung vorliegt, guter Ingenieurpraxis.

\section{Musterrechnungen}

In den Musterrechnungen wird vorausgesetzt, dass die seit Jahren in PLT-Schutzeinrichtungen eingesetzten Geräte betriebsbewährt sind. Aus der Sammlung der Stördaten bei der Namur seit 2002 wurden die Ausfallraten für bestimmte Gerätegruppen aus dem praktischen Einsatz ermittelt. Sie sind in der Tabelle 2 dargestellt.
Mit diesen Werten wurden die Musterrechnungen für verschiedene Redundanzgrade durchgeführt. Somit kann bei Verwendung betriebsbewährter Geräte nach NE 130 auf einen rechnerischen Einzelnachweis verzichtet werden; denn es ist bereits gerechnet! Diese Vorgehensweise ist mittlerweile auch in die VDI/VDE 2180, Blatt 4 [28] eingeflossen und erhält damit zusätzliches Gewicht. Eine weitere Effizienzsteigerung wäre möglich, wenn die Mannigfaltigkeit der eingesetzten Komponenten in PLT-Schutzeinrichtungen reduziert werden könnte, etwa durch Einführen von Standardgerätelisten. Hieran wird zurzeit auch auf Namur-Ebene intensiv gearbeitet.

\subsection{Nutzen der Stördatenanalyse}

Neben der firmenspezifischen Schwachstellenanalyse bietet die Störstatistik über die Sammlung der Daten bei der Namur auch übergeordnet erheblichen Nutzen. Wie wir wissen, sind Herstellerangaben zu Fehlerraten für Feldgeräte häufig konservativ. Daraus könnte die Forderung nach genereller Zweikanaligkeit von PLT-Schutzeinrichtungen abgeleitet werden. Mittels Stördatenanalyse konnte der Nachweis erbracht werden, dass die bisherige Vorgehensweise, bei kleineren Risiken einkanalig zu instrumentieren und bei höheren Risiken Redundanzen einzuführen, auch den quantitativen Anforderungen der neuen Normenwelt entspricht. Die Datenbasis beruht auf achtjähriger Beobachtung des Fehlerverhaltens von etwa 40000 Schutzeinrichtungen in rund 40 sich beteiligenden Firmen. Damit ist die Gefahr ungerechtfertigter Nachrüstkosten von mindestens 150 Millionen Euro für die Namur-Firmen gebannt. Das gemeinsame Ziel für die Wiederauferstehung einer vernünftigen Sicherheitstechnik sollte der Einsatz von betriebsbewährten Standardgeräten sein, die nach DIN 61508 (VDE 0803) entwickelt und in typischen (Redundanz-)Strukturen implementiert wurden und für die Musterrechnungen vorliegen. 


\begin{tabular}{|l|l|}
\hline \multicolumn{1}{|c|}{ Kanalart } & Kanalfehlerrrate für unerkannte passive Fehler $\lambda_{\text {Du }}$ \\
\hline Sensorik Messgröße P & $1 \cdot 10^{-6} \mathrm{~h}^{-1}=1000 \mathrm{FiT}$ \\
\hline Sensorik Messgröße T & $5 \cdot 10^{-7} \mathrm{~h}^{-1}=500 \mathrm{FiT}$ \\
\hline Sensorik Messgröße L & $4 \cdot 10^{-7} \mathrm{~h}^{-1}=400 \mathrm{FiT}$ \\
\hline Sensorik Messgröße F & $1 \cdot 10^{-6} \mathrm{~h}^{-1}=1000 \mathrm{FiT}$ \\
\hline Sensorik & $4 \cdot 10^{-7} \mathrm{~h}^{-1}=400 \mathrm{FiT}$ \\
\hline
\end{tabular}

TABELLE 2:

Richtwerte für sicherheitstechnische Kennzahlen von betriebsbewährten Geräten

$\mathrm{FiT}=$ Failures in time, d.h. Fehler pro $109 \mathrm{~h}$

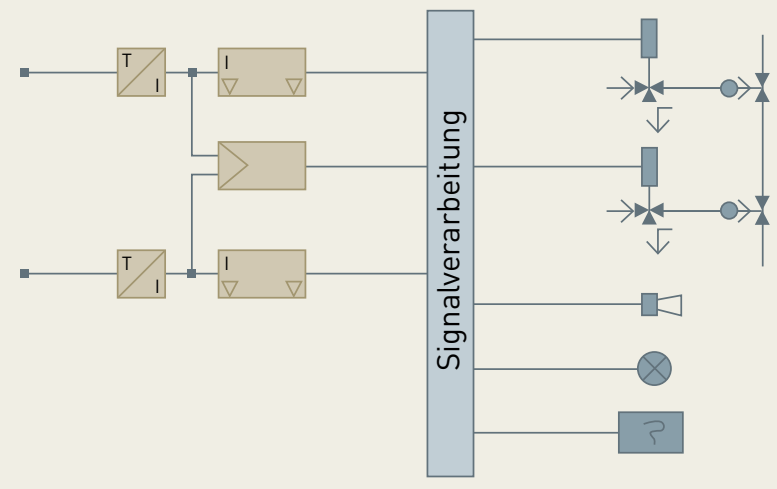

Anregeteil

Melde- und Auslöseteil
BILD 5:

Aufbau einer

PLT-Schutz-

einrichtung in

1v2-Bewertung

im Jahre 2010

\section{ZUSAMMENFASSUNG UND AUSBLICK}

Der Vergleich zwischen Bild 1 aus dem Jahr 1966 und Bild 5 aus dem Jahr 2010 könnte zu dem Schluss verleiten: Eigentlich hat sich in den letzten 45 Jahren nichts verändert. Sind deshalb die „Sicherheitsmacher“ stockkonservativ? Bei näherem Hinschauen stellt man jedoch fest, dass es seit 1966 deutliche Weiterentwicklungen, sowohl technischer, als auch organisatorischer Art, gegeben hat:

- Erweiterung des Schutzziels von ehemals nur „Anlage und Produkt“ auf „Personen und Umwelt“

- Klare Unterscheidung in „sicherheitsrelevante“ und „nicht sicherheitsrelevante“ Einrichtungen

- Orientierung der Auslegung am abzudeckenden Risiko

- Einbindung aller am Sicherheitsprozess Beteiligten in die Normungsaktivitäten

- Verbessertes Fehlerverhalten der Gerätetechnik und dank der Mikroprozessortechnik zahlreiche Diagnosemöglichkeiten

- Verbindungsprogrammierbare Steuerungen (VPS) wurden durch sicherheitsgerichtete speicherprogrammierbare Steuerungen (SSPS) abgelöst

- Die Zertifizierung von SSPS stellt sicher, dass ihre Hardware frei von passiven Fehlern ist

- Die Einführung von Sicherheitsmanagementsystemen regelt die Verantwortlichkeiten und zwingt zu systematischem Arbeiten
- Die Orientierung am Sicherheitslebenszyklus mit definierten Prüfschritten senkt die Fehlerrate in allen Phasen

- Die sorgfältige Dokumentation aller Schritte im Sicherheitsprozess sorgt für Transparenz und Nachvollziehbarkeit und schützt in einem Störungsfall vor dem Vorwurf der Fahrlässigkeit

- Flexibilisierung von Prüfzyklen

- Einführung des rechnerischen Nachweises der sicherheitstechnischen Verfügbarkeit

- Flächendeckende Einführung einer Stördatenerfassung und -analyse

Im Sicherheitsgeschäft gilt es, Änderungen behutsam, nach hinreichender Erprobung, einzuführen und rechtzeitig vor Übertreibungen und Fehlentwicklungen zu warnen. Wir haben nach Einschätzung des Autors ein sehr gutes PLT-Sicherheitskonzept in Deutschland, das auch im Zuge der Deregulierung ausgewogene Handlungsanweisungen an die beteiligten Gruppen gibt. Deshalb ist es auch glücklicherweise nicht erforderlich, wie in anderen Ländern zum Teil üblich, kleinlich bürokratisch und praxisfern überzureglementieren. Es geht darum, die Regelwerke auch tatsächlich umzusetzen und die gegebenen Freiheitsgrade unter „Einschaltung von Gehirn und ingenieurmäßigem Sachverstand" zu nutzen. Wir müssen deshalb unsere Kompetenz als Anwender selbstbewusst in die internationale Normung einbringen! Somit können wir darauf hinwirken, dass sich 
die Sicherheit nach Kochbuch in Grenzen hält. In anderen Ländern herrschen andere Rahmenbedingungen. Es seien nur das Stichwort „Produkthaftung“ und die Angst vor exorbitant hohen Regressforderungen genannt. Daran ausgerichtete Vorgehensweisen weltweit in ganz anderen Rechtsräumen und gewachsenen Kulturen einführen zu wollen, führt lediglich zu überflüssigem Aktionismus, ohne die Sicherheit der Anlagen zu verbessern. Dazu gehört aber zwangsläufig auch, dass Unternehmen der Anwenderseite bereit sind, unsere Normungsaktivitäten finanziell zu unterstützen. Aussagen wie „Lasst uns mal sportlich $10 \%$ in der Regelwerksverfolgung und der Gremienarbeit einsparen“ sind das falsche Signal und gehören in die Steinzeit! Zurzeit bringt die deutsche Delegation bei der anstehenden Überarbeitung der IEC 61511 folgende Themen ein:

- Betriebsbewährte Geräte (NE 130)

- Vorgehen bei elektromechanischen Komponenten

- Mitbenutzung von PLT-Betriebseinrichtungen und

- Nutzbare Lebensdauer von Geräten

Es wird Kompromisse geben müssen, aber die Ergebnisse werden der Sicherheitsevolution sicher zuträglich sein.

\section{AUTOR}

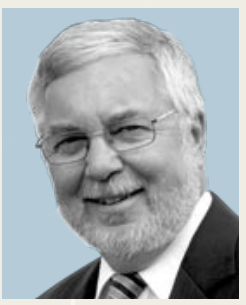

\section{DR. PIRMIN NETTER}

(geb. 1949) studierte Experimentalphysik in Heidelberg. Von 1981 bis 1996 hatte er verschiedene Positionen in der EMRBetriebsbetreuung der Hoechst AG inne. Von 1997 bis 2009 war er Leiter der Sicherheitsüberwachung Hoechst AG beziehungsweise Abt. Arbeitsschutz und Anlagensicherheit der Infraserv Höchst. Sein Hauptarbeitsgebiet ist Anlagensicherung mit Mitteln der Prozessleittechnik. Dr. Netter ist Mitglied in mehreren nationalen Gremien. Bis 2010 war er deutscher Sprecher im IEC SC65A. Er ist bekannt gegebener Sachverständiger gem. § 29a BImschG.

Infraserv GmbH \& Co. Höchst KG, Industriepark Höchst, C 769, D-65926 Frankfurt am Main, Tel. +49 (0) 693056498 E-Mail: Pirmin.Netter@infraserv.com

\section{REFERENZEN}

[1] VDI/VDE-Richtlinie 2180, Blatt 1-7: Sicherung von Anlagen der Verfahrenstechnik, 1966ff

[2] Störfallverordnung: Zwölfte Verordnung zur Durchführung des Bundes-Immissionsschutzgesetzes,1980

[3] G. Strohmann: Anlagensicherung mit Mitteln der MSR-Technik,1983

[4] Hölscher/Rader: Mikrocomputer in der Sicherheitstechnik, 1984

[5] VDI/VDE-Richtlinie 2180, Blatt 1-5: Sicherung von Anlagen der Verfahrenstechnik mit Mitteln der MSR-Technik, $1984 \mathrm{ff}$

[6] TRbF 510: Richtlinie/Bau- und Prüfgrundsätze für Überfüllsicherungen, 1985

[7] DIN VDE 31000 Teil 2: Allgemeine Leitsätze für das sicherheitsgerechte Gestalten technischer Erzeugnisse, 1987

[8] DIN V 19250: Grundlegende Sicherheitsbetrachtungen für MSR-Schutzeinrichtungen, 1989

[9] DIN V 19251: MSR-Schutzeinrichtungen, Anforderungen und Maßnahmen zur gesicherten Funktion, 1995

[10] AD-Merkblatt A6: Sicherheitseinrichtungen gegen Drucküberschreitung, MSR-Sicherheitseinrichtungen, 1986

[11] Zwölfte Verordnung zur Durchführung des BundesImmissionsschutzgesetzes (Störfall-Verordnung) 12. BImSchV- Novelle 1988

[12] DIN V VDE 0801: Grundsätze für Rechner in Systemen mit Sicherheitsaufgaben, 1990

[13] NE 31: Anlagensicherung mit Mitteln der Prozessleittechnik, 1993

[14] DIN V VDE 0801/A1: Grundsätze für Rechner in Systemen mit Sicherheitsaufgaben, 1994

[15] VdTÜV-Merkblatt 372: Leitlinie für die Prüfung sicherheitsrelevanter MSR-Einrichtungen in Anlagen, 1997

[16] VDI/VDE-Richtlinie 2180, Blatt 1-5: Sicherung von Anlagen der Verfahrenstechnik mit Mitteln der Prozessleittechnik, 1998

[17] Bundesministerium für Umwelt, Naturschutz und Reaktorsicherheit: Vollzugshilfe zur Störfallverordnung, 2004

[18] NE 79: Mikroprozessorbestückte Geräte in der Anlagensicherung, 1999

[19] VDI/VDE-Richtlinie 2180, Blatt 5: Einsatz von speicherprogrammierbaren Steuerungen, 2000

[20] NE 93: Nachweis der sicherheitstechnischen Zuverlässigkeit von PLT-Schutzeinrichtungen, 2001

[21] NE 97: Feldbus für Sicherungsaufgaben, 2003

[22] NE 106: Prüfintervalle für PLT-Schutzeinrichtungen, 2006

[23] DIN EN 61508 (VDE 0803): Funktionale Sicherheit sicherheitsbezogener elektrischer / elektronischer / programmierbar elektronischer Systeme, 2002

[24] DIN EN 61511 (VDE 0810): Funktionale Sicherheit - Sicherheitstechnische Systeme für die Prozessindustrie, 2005

[25] VDI/VDE-Richtlinie 2180, Blatt 1-5: Sicherung von Anlagen der Verfahrenstechnik mit Mitteln der Prozessleittechnik, 2007-2010

[26] NE 126: Bestandsschutz für PLT-Schutzeinrichtungen, 2009

[27] NE 130: Betriebsbewährte Geräte für PLT-Schutzeinrichtungen und vereinfachte SIL-Berechnung, 2010

[28] VDI/VDE-Richtlinie 2180, Blatt 4: Nachweis der Hardware-Sicherheitsintegrität einer PLT-Schutzeinrichtung, 2010 